\title{
PRIMITIVE ELEMENTS AND ONE RELATION ALGEBRAS(1)
}

BY

\section{CATHERINE AUST}

ABSTRACT. Let $F$ be a free algebra in a variety $V$. An element $p$ of $F$ is called primitive if it is contained in some free generating set for $F$. In 1936, J. H. C. Whitehead proved that a group with generators $g_{1}, \ldots, g_{n}$ and one relation $r=1$ is free if and only if the relator $r$ is primitive in the free group on $g_{1}, \cdots, g_{n}$. In this paper, the question of whether there is an analogous theorem for other varieties is considered. A necessary and sufficient condition that a finitely generated, one relation algebra be free is proved for any Schreier variety of nonassociative linear algebras and for any variety defined by balanced identities. An identity $u\left(x_{1}, \ldots, x_{n}\right)=v\left(x_{1}, \ldots, x_{n}\right)$ is called balanced if each of $u$ and $v$ has the same length and number of occurrences of each $x_{i}$. General sufficiency conditions that a finitely generated, one relation algebra be free are given, and all of the known results analogous to the Whitehead theorem are shown to be equivalent to a general necessary condition. Also an algebraic proof of Whitehead's theorem is outlined to suggest the line of argument for other varieties.

1. Introduction. Let $F$ be a free algebra in a variety $V$. We say that $p$ is primitive in $F$ if there is some free generating set for $F$ which contains $p$. In 1936, J. H. C. Whitehead proved that a group with generators $g_{1}, \cdots, g_{n}$ and one relation $r=1$ is free if and only if the relator $r$ is primitive in the free group on $g_{1}, \cdots, g_{n}[14]$. In 1970, G. P. Kukin proved a similar result for one relation Lie algebras [8]. We raise the following question: For which varieties $V$ can one find a necessary and sufficient condition that a finitely generated, one relation $V$-algebra be free? In free groups, there is an algorithm for deciding when an element is primitive. Combined with Whitehead's result this gives a solution

Presented to the Society, November 11, 1973; received by the editors February 26, 1973. AMS (MOS) subject classifications (1970). Primary 08A10, 08A25; Secondary 17A99, $20 \mathrm{M} 05$.

Key words and phrases. Primitive element, free algebra, one relation algebra, elementary transformation, Schreier variety of nonassociative linear algebras, balanced identity, balanced variety.

(1) This work was done as part of a doctoral dissertation at Emory University prepared under the supervision of Professor Trevor Evans. The author is indebted to him for many valuable suggestions. 
to one case of the isomorphism problem. Since it is not known when two one relation groups are isomorphic [11, p. 401], this special case is a significant result. In other varieties where there is an algorithm for deciding whether an element is primitive, a condition that a one relation algebra be free might provide a similar solution for one case of the isomorphism problem.

In this paper, we begin an investigation of the above question. We prove a general sufficiency condition $(\$ 3)$ and indicate a general necessary and sufficient condition which we show to be equivalent to all the known results. We outline a proof of Whitehead's theorem $(\$ 4)$, since no algebraic proof is available in the literature, and prove a necessary and sufficient condition for any Schreier variety of nonassociative linear algebras $(\$ 5)$ and any variety defined by "balanced" identities (\$6). We also indicate the other varieties for which there is a theorem.

We have also proved a theorem like the Whitehead theorem for the varieties of loops and quasigroups. The proofs require the development of Nielson transformations for loops and quasigroups and will be given in a future paper devoted to automorphisms and primitive elements in these algebras.

2. Preliminary ideas. By an algebra $\mathfrak{Q}=(A, \Omega)$ we mean a nonempty set $A$ together with a nonempty set $\Omega$ of finitary operations. We usually denote an algebra by its underlying set. We use the concepts of word, subword (or component), elementary transformation, and algebra given by generators and relations as in [3]. Thus, if $\left\{f_{j}: j \in J\right\}$ is a set of operation symbols, we define a word in the symiols $x_{1}, x_{2}, \ldots$ as follows:

(i) each $x_{i}$ is a word, and

(ii) if $f_{j}$ represents an $n$-ary operation and $u_{1}, \ldots, u_{n}$ are words the $x_{i}$ 's, then $f_{j}\left(u_{1}, \ldots, u_{n}\right)$ is a word.

We use the notations $u(\mathbf{x}), u\left(x_{1}, \ldots, x_{m}\right)$ to denote that $u$ is a word in $x_{1}, x_{2}, \ldots$, in at most $x_{1}, \ldots, x_{m}$, respectively. In the construction of the algebra $A$ in the variety $V$ given by generators $g_{1}, g_{2}, \ldots$ and relations $r_{\lambda}(g)=s_{\lambda}(g), \lambda \in \Lambda$, an elementary transformation of a word $w$ in the $g_{i}$ 's is one of the following:

(i) replacing one occurrence of the subword $u\left(w_{1}, \ldots, w_{m}\right)$ in $w$ by the word $v\left(w_{1}, \ldots, w_{m}\right)$, where $w_{1}, \ldots, w_{m}$ are words in the $g_{i}$ 's and $u\left(x_{1}, \ldots, x_{m}\right)=$ $v\left(x_{1}, \ldots, x_{m}\right)$ or $v(\mathbf{x})=u(\mathbf{x})$ is a defining identity for $V$,

(ii) replacing an occurrence of the subword $r_{\lambda}(\mathrm{g})$ in $w$ by the word $s_{\lambda}(\mathrm{g})$, or vice versa.

Two words in $g_{1}, g_{2}, \ldots$ are equivalent if there is a finite sequence of elementary transformations changing one into the other. This definition yields an equivalence relation on all words in $g_{1}, g_{2}, \ldots ;$ the equivalence classes are the elements of $A$. 
3. A universal sufficiency condition. For each of the theorems analogous to the Whitehead theorem, proving the necessity of the given condition depends heavily on properties of the particular variety involved. On the other hand, it is easy to obtain a sufficiency condition that holds for every variety.

The following condition is a simple generalization of the group condition.

Theorem 1. Let $V$ be a variety and $F$ be the free algebra in $V$ on $g_{1}, \cdots, g_{n}$. If $\left\{p_{1}, \cdots, p_{m}\right\}$ is a $V$-free generating set for $F$ and $u\left(x_{1}, \ldots, x_{m-1}\right)$ is $a V$-word, then an algebra $A$ in $V$ presented by

$$
\left\langle g_{1}, \cdots, g_{n} ; p_{j}=w\left(p_{1}, \cdots, p_{i-1}, p_{j+1}, \cdots, p_{m}\right)\right\rangle
$$

is free in $V$.

Proof. Since $F$ is freely generated by $p_{1}, \ldots, p_{m}, A$ has a presentation $\left\langle p_{1}, \cdots, p_{m} ; p_{j}=w\left(p_{1}, \ldots, p_{j-1}, p_{j+1}, \ldots, p_{m}\right)\right\rangle$. Removing $p_{j}$ and the relation $p_{j}=w$, we see that $A$ if free on $p_{1}, \ldots, p_{j-1}, p_{j+1}, \ldots, p_{m}$.

In the case of certain varieties such as groups, rings, and loops, which have special one element subalgebras $\{e\}$, any relation is equivalent to a relation equating a word to $e$, and relations are usually given in this way. In these varieties $e$ can be expressed as a word in any set of elements from an algebra. Thus, the group, ring, loop, etc., presented by $\left\langle g_{1}, \ldots, g_{n} ; p=e\right\rangle$ (for these special varieties it is customary to write only the left-hand side of relations of the form $r=e$ and, hereafter, we adopt this convention) is free if $p$ is primitive in the free algebra on $g_{1}, \ldots, g_{n}$. Thus, Theorem 1 does give a generalization of one-half of the group theorem.

The following corollary to Theorem 1 gives a more general sufficiency condition and includes all the known results.

Corollary 1. Let $V$ be a variety and $F$ the free algebra in $V$ on $g_{1}, \ldots, g_{n}$. An algebra $A$ in $V$ presented by $\left\langle g_{1}, \ldots, g_{n}: r(g)=s(g)\right\rangle$ is $V$-free if there exists a $V$ word $w\left(x_{1}, \ldots, x_{m-1}\right)$ and a free generating set $\left\{p_{1}, \ldots, p_{m}\right\}$ for $F$ such that the relation $p_{m}=w\left(p_{1}, \ldots, p_{m-1}\right)$ generates exactly the same congruence on $F$ as the relation $r=s$.

In each of the varieties for which there is a theorem stating a necessary and sufficient condition that a one relation algebra be free, the theorem is equivalent to Corollary 1 plus its converse. This equivalence is not always evident, because, in some cases, one can say much more about words $p_{1}, \cdots, p_{m}, r$, and $s$ which satisfy the hypothesis of Corollary 1 . In these cases, we indicate how to obtain the equivalence.

There are varieties in which the $m$ and $n$ of Theorem 1 and Corollary 1 can be any positive integers. The variety of Jónsson-Tarski algebras is one [7]. All 
the finitely generated free algebras in this variety are isomorphic. Thus, the free algebras on $n$ generators has a free generating set containing $m$ elements, for every positive integer $m$.

4. Abelian groups and groups. The impetus for this investigation was an attempt to give an algebraic proof of Whitehead's theorem. Whitehead's proof uses topological methods. The theorem is stated without proof on p. 167 of [11] (although part occurs in Exercise 20 of $\$ 4.4$ ). In this section we outline our algebraic proof, since the line of argument suggests an approach for other varieties. We write all groups multiplicatively.

The following results are needed for our proof of the Whitehead theorem. Theorem 2 gives the condition that a finitely generated, one relator abelian group be free. It can be proved using elementary properties of free abelian groups. Theorem 4 is a deep result from combinatorial group theory.

Theorem 2. Let $A$ be an abelian group presented by

$$
\left\langle a_{1}, \ldots, a_{n} ; a_{1}^{k_{1}} a_{2}^{k_{2}} \ldots a_{n}^{k_{n}}\right\rangle \text {, }
$$

where at least one of $k_{1}, \ldots, k_{n} \neq 0$. Then $A$ is free abelian if and only if $a_{1}^{k_{1}} \ldots a_{n}^{k_{n}}$ is primitive in the free abelian group $F$ generated by $a_{1}, \ldots, a_{n}$. Moreover, if $A$ is free, $A$ is free on $n-1$ generators.

Theorem 3. Let $F$ be the free group on $g_{1}, \ldots, g_{n}$ and $H$ a normal sub. group of $F$. If $F / H$ is a free group, then there exists a free generating set $\left\{p_{1}, \cdots, p_{n}\right\}$ for $F$ such that $H$ is the normal subgroup generated by $p_{1}, \cdots, p_{k}$, $k \leq n[11$, Theorem 3.3, p. 132].

Theorem 4. Let $F$ be the free group on $g_{1}, \ldots, g_{n}$. The words $r\left(g_{1}, \ldots, g_{n}\right)$ and $s\left(g_{1}, \ldots, g_{n}\right)$ generate the same normal subgroup of $F$ if and only if $r$ is conjugate to $s$ or $s^{-1}[11$, Theorem 4.11, p. 261].

We now indicate a proof of Whitehead's theorem.

Theorem 5 (Whitehead). Let $G$ be a group presented by $\left\langle g_{1}, \ldots, g_{n}\right.$; $\left.r\left(g_{1}, \ldots, g_{n}\right)\right\rangle$, where $r(\mathrm{~g})$ is a nontrivial reduced word. Then, $G$ is a free group if and only if $r(\mathrm{~g})$ is primitive in the free group $F$ generated by $g_{1}, \cdots, g_{n}$.

Proof. As discussed earlier, Theorem 1 gives the implication one way. We need to prove that, if $G$ is free, then $r$ is primitive.

(i) $G \cong F / R$, where $R$ is the normal subgroup of $F$ generated by $r$. By Theorem 3, there is a free generating set $\left\{p_{1}, \ldots, p_{n}\right\}$ for $F$ such that $R$ is the normal subgroup generated by $\left\{p_{1}, \ldots, p_{k}\right\}$. Then, $F / R$ is freely generated by $p_{k+1} R, \cdots, p_{n} R$. 
(ii) Since $r$ is a nontrivial word, $k \geqq 1$. We show $k=1$ by considering $A=(F / R) /(F / R)^{\prime}$. Since $F / R$ is a free group on $n-k$ generators, $A$ is free abelian on $n-k$ generators. Now, $A=(F / R) /\left(F^{\prime} R / R\right) \cong F / F^{\prime} R \cong\left(F / F^{\prime}\right) /\left(F^{\prime} R / F^{\prime}\right)$, $F / F^{\prime}$ is a free abelian group on $n$ generators, and $F^{\prime} R / F^{\prime}$ is the subgroup of $F / F^{\prime}$ generated by the coset $r F^{\prime}$. Since $A$ is not isomorphic to $F / F^{\prime}, r F^{\prime}$ is a nonzero element of $F / F^{\prime}$. Then, we may apply Theorem 2 to $A$ to obtain that $A$ is free on $n-1$ generators. Since all free generating sets for $A$ must have the same number of elements, $k=1$.

(iii) Then $r$ generates the same normal subgroup as the primitive element $p_{1}$. By Theorem $4, r=t^{-1} p_{1}^{\delta} t$, where $\delta=1$ or -1 . Since $r$ is the image of the primitive element $p_{1}^{\delta}$ under an inner automorphism of $F, r$ is primitive.

Using Theorem 4 as in (iii) above, one can prove that an element in a free group generates the same normal subgroup as a primitive element if and only if it is primitive. This result shows that Whitehead's theorem is equivalent to Corollary 1 and its converse, in the case of groups. A similar line of argument yields that Theorem 2 is equivalent to Corollary 1 plus its converse for abelian groups.

5. Nonassociative linear algebras. G. P. Kukin has proved a theorem like the Whitehead theorem for Lie algebras [8]. His result: A finitely generated, one relator Lie algebra is free if and only if the relator is primitive in the corresponding free Lie algebra.

A Lie algebra is a nonassociative linear algebra over a field. The class of nonassociative linear algebras over a fixed field $\Phi$ may be regarded as a variety if scalar multiplication by a field element is taken to be a unary operation. We now prove results similar to Kukin's for several different varieties of nonassociative linear algebras.

A variety is Schreier if any subalgebra of a free algebra is also free. In [10] J. Lewin describes the automorphisms of the finitely generated free algebras in the variety $V$, where $V$ is any Schreier subvariety of the variety of all nonassociative linear algebras with identity element 1 over an infinite field $\Phi$. Using his results, we prove a necessary and sufficient condition for a finitely generated, one relator $V$-algebra to be free.

In our proof we follow Lewin's terminology and presentation of free algebras. Let $V_{0}$ be the variety of all nonassociative linear algebras over $\Phi$ with identity element 1 . Let $F_{0}$ be the free algebra in $V_{0}$ on $a_{1}, \ldots, a_{n}$, and let $F$ be the free algebra in $V$ on $p_{1}, \ldots, p_{n}$. The monomials of $F_{0}$ are the elements of $G \cup\{1\}$, where $G$ is the free groupoid on $a_{1}, \ldots, a_{n}, M=G \cup\{1\}$ is a basis for $F_{0}$ over $\Phi$. Let $H^{i}$ denote the subspace of $F_{0}$ generated by the monomials of degree $i$. Then, $H^{i} H^{j} \subseteq H^{i+j}$, and $F_{0}=\Sigma_{i=0}^{\infty} H^{i}$. Let $\eta$ be the homomorphism 
of $F_{0}$ onto $F$ defined by $a_{i} \eta=p_{i}, i=1,2, \ldots, n$, and $N=\operatorname{Ker} \eta$. Then, $F \simeq$ $F_{0} / N$ under the correspondence $w\left(a_{1}, \ldots, a_{n}\right)=w\left(p_{1}, \ldots, p_{n}\right)$, and $N$ is a fully invariant ideal in $F_{0}$. By Proposition 2 in [10], $N$ is generated by a homogeneous set of elements. Hence, for each $w$ in $F_{0}, w$ belongs to $N$ if and only if each homogeneous term of $w$ belongs to $N$. The elements of $M \eta$ are monomials in $F$, and there is a subset $B$ of $M \eta$ such that $B$ is a basis for $F$ over $\Phi$. The elements of $H^{i} \eta$ are the homogeneous elements of degree $i, B \cap H^{i} \eta$ is a basis for $H^{i} \eta$, and $F=\Sigma_{i=0}^{\infty} H^{i} \eta$.

Theorem 6. Let $V$ be a nontrivial Schreier subvariety of the variety $V_{0}$ of all nonassociative linear algebras with identity element 1 over a infinite field $\Phi$, Let $A$ be an algebra in $V$ presented by $\left\langle g_{1}, \ldots, g_{n} ; r\left(g_{1}, \ldots, g_{n}\right)\right\rangle$, where $r(g)$ is a nontrivial $V$-word, and let $F$ be the free $V$-algebra generated by $g_{1}, \ldots$, $g_{n}$. Then, $A$ is $V$-free if and only if $r(g)$ generates the same ideal in $F$ as that generated by some primitive element.

Proof. If $r$ generates the same ideal in $F$ as some primitive element $p$, the relation $r=0$ generates the same congruence in $F$ as the relation $p=0$. Then, by Corollary $1, A$ is free.

To show that, if $A$ is free, then $r$ generates the same ideal as a primitive element, we consider $F / R \cong A$, where $R$ is the ideal of $F$ generated by $r$. By Theorem 5 of [10], there exists a $V$-free set of generators $p_{1}, \cdots, p_{n}$ for $F$ such that $R$ is the ideal generated by $p_{1}, \ldots, p_{k}, k \leq n$. Since $r$ is a nontrivial word, $R \neq\{0\}$. Thus, $k \geq 1$. As in the group theorem, we show that $k=1$ and, thus, $r$ generates the same ideal as the primitive element $p_{1}$. For the remainder of the proof, we regard the elements of $F$ as words in $p_{1}, \ldots, p_{n}$.

Let $H^{i}, \eta$, and $N$ be as in the paragraph preceding the theorem. Let $K$ denote the ideal $\Sigma_{i=2}^{\infty} H^{i} \eta$. We show that $\left\{1+K, p_{1}+K, \ldots, p_{n}+K\right\}$ is a basis for the vector space $F / K$. In $F_{0},\{1\}$ is a basis for $H^{0}$ and $\left\{a_{1}, \ldots, a_{n}\right\}$ is a basis for $H^{1}$. Since $V$ is a nontrivial variety, $1 \neq 0$ in $F$. Then, $\{1 \eta=1\}$ is a basis for $H^{0} \eta$. Some subset of $\left\{p_{1}=a_{1} \eta, \ldots, p_{n}=a_{n} \eta\right\}$ is a basis for $H^{1} \eta$. We need only show that $p_{1}, \ldots, p_{n}$ are linearly independent to establish that the basis is $\left\{p_{1}, \ldots, p_{n}\right\}$. If $\sum_{i=1}^{n} a_{i} p_{i}=0$ for some $a_{1}, \ldots, a_{n}$ in $\Phi$, $\left(\sum_{i=1}^{n} a_{i} a_{i}\right) \eta=0$. Then, $\sum_{i=1}^{n} a_{i} a_{i}$ is in $N$. Suppose $a_{j} \neq 0$ for some $j, 1 \leq j$ $\leq n$, and let $\epsilon$ be the endomorphism of $F_{0}$ defined by $a_{j} \epsilon=a_{j}^{-1} a_{j}$ and $a_{i} \epsilon=0$ for $i \neq j$. Since $N$ is fully invariant, $a_{j}=\left(\sum_{i=1}^{n} a_{i} a_{i}\right) \epsilon$ is in $N$. Then, $p_{j}=a_{j} \eta=0$ in $F$ and $F$ is trivial. This is a contradiction.

We now consider the subspace $R+K / K$ of the quotient space $F / K . R$ is generated as an ideal of $F$ by $p_{1}, \ldots, p_{k}$. Thus, for any $w$ in $R, w=\sum_{i=1}^{m} a_{i} b_{i}$, where each $\alpha_{i} \neq 0$ and each $b_{i}$ is a monomial of $F$ involving one of $p_{1}, \cdots, p_{k}$. 
Then, the homogeneous term of least degree in $w$ has degree 1 , and $w+K=$ $\left(\sum_{i=1}^{k} \delta_{i} p_{i}\right)+K=\sum_{i=1}^{k} \delta_{i}\left(p_{i}+K\right)$, for some $\delta_{1}, \ldots, \delta_{k}$ in $\Phi$. Hence, $R+K / K$ is the subspace of $F / K$ generated by $\left\{p_{1}+K, \ldots, p_{k}+K\right\}$ and has dimension $k$.

Since $R$ is the ideal of $F$ generated by $r$, if $w$ is in $R, w=\sum_{i=1}^{m} w_{i}$, where each $w_{i}$ is a product of elements of $F$ at least one of which is $r$. Then, $w+K=$ $\left(\sum_{i=1}^{m} w_{i}\right)+K=\sum_{i=1}^{m}\left(w_{i}+K\right)$. From the preceding discussion, $r$ has degree greater than or equal to 1 . Since each $w_{i}$ is a product involving $r$, for each $i=1, \ldots, m, w_{i}+K=\beta_{i} r+K$ for some $\beta_{i}$ in $\Phi$. Let $\beta=\sum_{i=1}^{m} \beta_{i}$. Then, $w+K=\sum_{i=1}^{m}\left(\beta_{i} r+K\right)=\beta(r+K)$. Then, $R+K / K$ is contained in the subspace of $F / K$ generated by $r+K$. Since $\alpha(r+K)=\alpha r+K$ is in $R+K / K$ for any $a$ in $\Phi, R+K / K$ is the subspace of $F / K$ generated by $r+K$. Then, $R+K / K$ has dimension 0 or 1 as $r$ is or is not contained in $K$. Since the dimension of $R+K / K$ is unique, it must be that $k=1$ and is the dimension.

As Lewin implies in his introduction to [10], his results also hold in the variety of all nonassociative linear algebras (without identity) over an infinite field $\Phi$. Only slight alterations are required to adapt the proofs in [10] and the proof of Theorem 6 to obtain the following:

Theorem 7. Let $W$ be a nontrivial Schreier subvariety of the variety $W_{0}$ of all nonassociative linear algebras over an infinite field $\Phi$. Let $A$ be an algebra in $W$ presented by $\left\langle g_{1}, \ldots, g_{n}: r\left(g_{1}, \ldots, g_{n}\right)\right\rangle$, where $r(g)$ is a nontrivial W-word, and let $F$ be the free W-algebra generated by $g_{1}, \ldots, g_{n}$. Then, $A$ is W-free if and only if $r(\mathrm{~g})$ generates the same ideal in $F$ as that generated by some primitive element.

Several subvarieties of $W_{0}$ have been proved to be Schreier. They are the varieties of all commutative algebras (satisfying the identity $x y=y x$ ) [13], anticommutative algebras (satisfying $x y+y x=0$ ) [13], Lie algebras [12] and [15], and $W_{0}$ itself [9]. $V_{0}$ [9] and the variety of commutative nonassociative linear algebras with identity [13] are Schreier subvarieties of $V_{0}$. We note that applying Theorem 7 to Lie algebras gives a weaker result than Kukin's because of our restriction to an infinite field.

Given the special way in which relations are presented for linear algebras, it is easy to see that Theorems 6 and 7 are equivalent to Corollary 1 plus its converse for the varieties involved. The equivalence of Kukin's result and Corollary 1 plus its converse follows from the fact that an element in a free Lie algebra generates the same ideal as a primitive element if and only if it is primitive. The latter follows from Theorem 1 and Proposition I of [8].

6. A return to universal algebra. In proving Theorem 6 , we used well-known results concerning vector spaces. The following results concerning vector spaces are also well-known: 
(i) Every vector space has a basis.

(ii) Every nonzero element is contained in a basis.

Restating these two results in the language of free algebras, we have (i) every vector space is a free vector space and (ii) every nonzero element of a vector space is primitive. In this situation, the question of a necessary and sufficient condition that a finitely generated one relation algebra be free becomes trivial. Other varieties in which every algebra is free and every nonzero element is primitive are the varieties of abelian groups of prime exponent, right zero semigroups, and left zero semigroups. These varieties are equationally complete [6]. In many equationally complete varieties, every algebra is free, and, thus, the question of an analog of the Whitehead theorem is trivial. In the variety of constant semigroups (semigroups satisfying $x y=z w$ )-another equationally complete variety-the question is also easily answered. Here every nontrivial algebra is free, but the one element algebra is not [5]. If we consider only nontrivial relations on the constant semigroup generated by $g_{1}, \ldots, g_{n}$-such relations are necessarily of the form $g_{i}=$ $w\left(g_{1}, \ldots, g_{n}\right)$-we see that any one generator, one relation constant semigroup is trivial and any one relation constant semigroup on two or more generators is free.

We now consider a type of variety for which one can obtain a theorem like the Whitehead theorem. Two algebras are said to have the same operation, or similarity type, if they have the same number of $n$-ary operations for each nonnegative integer $n$. Let $W$ be the variety of all algebras of some fixed operation type (the free algebras in $W$ are word, or Peano, algebras [2]). The free algebra $F(W)$ in $W$ generated by the countably infinite set of generators $\left\{x_{1}, x_{2}, \ldots,\right\}$ consists of all $W$-words in the $x_{i}{ }^{\prime}$ s. Distinct words in $F(W)$ are distinct elements. Let $V$ be a subvariety of $W$ defined by a set of identities $I$. We consider an identity in $I$ to be an equation $u\left(x_{1}, \ldots, x_{m}\right)=v\left(x_{1}, \ldots, x_{m}\right)$, where $u$ and $v$ are words in $F(W)$. Let $F(V)$ denote the free algebra in $V$ on a countably infinite set of generators. From universal algebra we have that $F(V)$ is isomorphic to $F(W) / \theta_{l}$, where $\theta_{l}$ is the fully invariant congruence on $F(W)$ generated by the set of all ordered pairs $(u, v)$ such that $u=v$ is in $I$. The closure of $I$, denoted $\mathrm{Cl}(I)$ is the set of all identities satisfied by all the algebras in $V$. From universal algebra, $u=v$ is in $\mathrm{Cl}(l)$ if and only if $(u, v)$ is in $\theta_{I^{\circ}}$.

We define the length of a word $w\left(x_{1}, \ldots, x_{n}\right)$ in $F(W)$ to be the total number of generators and operation symbols occurring in it; the length of $w$ will be denoted by $|w|$. An identity $u=v$ in $I$ is called balanced if $|u|=|v|$ and each $\boldsymbol{x}_{i}$ occurs the same number of times in each of $u$ and $v$. (This is a generalization of Belousov's definition of a balanced quasigroup identity [1].) If each of the identities in $I$ is balanced, we call $I$ a balanced set of identities and $V$ a balanced variety. 
Lemma 1. Let I be a set of identities. If I is balanced, then $\mathrm{Cl}(I)$ is balanced.

Proof. Let $\Psi=\{(u, v): u=v$ is in $I\}$ and $\Delta=\{(u, u): u$ is in $F(W)\}$. Then, let $\theta_{0}=\Psi \cup \Psi^{-1} \cup \Delta$, and, for each $i=1,2, \ldots$,

$$
\begin{aligned}
& \theta_{3 i-2}=\left\{(u, v):(u, w) \text { and }(w, v) \text { are in } \theta_{3 i-3} \text { for some } w \text { in } F\right\}, \\
& \theta_{3 i-1}=\theta_{3 i-2} \cup\left\{\left(f\left(u_{1}, \ldots, u_{t}\right), f\left(v_{1}, \ldots, v_{t}\right)\right): f \text { is a } t\right. \text {-ary } \\
& \left.\quad \text { on } F(W) \text { and }\left(u_{i}, v_{i}\right) \text { is in } \theta_{3 i-2} \text { for } i=1, \ldots, t\right\},
\end{aligned}
$$

and

$$
\begin{array}{r}
\theta_{3 i}=\theta_{3 i-1}-\left\{\left(u\left(q_{1}, \cdots, q_{m}\right), v\left(q_{1}, \ldots, q_{m}\right)\right):\left(u\left(x_{1}, \ldots, x_{m}\right),\right.\right. \\
\left.v\left(x_{1}, \ldots, x_{m}\right)\right) \text { is in } \theta_{3 i-1} \text { and } q_{i} \text { is in } F(W), \\
\text { for } i=1, \cdots, m\} .
\end{array}
$$

Then, $\theta_{I}=\bigcup_{i=0}^{\infty} \theta_{i}$ is the fully invariant congruence of $F(W)$ generated by $\theta$, and $\mathrm{Cl}(I)$ is the set of all equations $u=v$ such that $(u, v)$ is in $\theta_{I}$. Using induction on $n$, for every $(u, v)$ in $\theta_{n}, u=v$ is a balanced identity.

Lemma 2. If the variety $V$ is defined by each of the sets of identities $I_{1}$ and $I_{2}$, and $I_{1}$ is balanced, then $I_{2}$ is balanced.

Proof. By Lemma $1, \mathrm{Cl}\left(I_{1}\right)$ is balanced. Since each of $I_{1}$ and $I_{2}$ define $V, \mathrm{Cl}\left(I_{1}\right)=\mathrm{Cl}\left(I_{2}\right)$. Since $I_{2} \subseteq \mathrm{Cl}\left(I_{2}\right)$, each identity in $I_{2}$ must be balanced.

By Lemma 2, whether a variety is balanced is independent of the particular set of defining identities one considers.

Lemma 3. If $V$ is a balanced variety and $U$ is a variety containing $V$, then $U$ is balanced.

Proof. Let $I$ and $J$ be sets of identities which define $V$ and $U$, respectively. Then, $\mathrm{Cl}(J) \subseteq \mathrm{Cl}(I)$ and $\mathrm{Cl}(I)$ is balanced. Since $J \subseteq \mathrm{Cl}(J), U$ is defined by a balan_ed set of identities.

For any similarity type, the variety of all algebras of that similarity type is a balanced variety. The variety $\mathcal{G}$ of all groupoids, algebras with one binary operation, is perhaps the most familiar of these. Other familiar balanced varieties are the varieties $\mathcal{S}$ of all semigroups (groupoids satisfying $x(y z)=(x y) z)$ and $\mathcal{C}$ of all commutative semigroups (semigroups satisfying $x y=y x$ ). Since $\mathcal{C}$ is a subvariety of $\mathcal{S}$, and $\mathcal{S}$ is a subvariety of $\mathcal{G}$, any subvariety of $\mathcal{G}$ which contains $\mathcal{S}$ or $\mathcal{C}$ is also a balanced variety. 
We shall prove a theorem like the Whitehead theorem for any balanced variety of finite similarity type, that is, a balanced variety such that each algebra in the variety has only a finite number of operations. First, we develop some properties of the finitely generated free algebras in such a variety. For any balanced variety $V$, we consider the free algebra generated by a set $g_{1}, g_{2}, \ldots$ to consist of equivalence classes of words in the $g_{i}$ 's. The length of a word $w$ in the $g_{i}$ 's, denoted by $|w|_{g}$ is defined analogously as above.

The following lemma establishes a basic property that we use several times.

Lemma 4. Let $V$ be a balanced variety and $F$ be the free algebra in $V$ on $g_{1}, g_{2}, \ldots$. Let $u$ and $v$ be words in the $g_{i}$ 's which are equivalent in $F$. Then, $u$ and $v$ bave the same length and the same number of occurrences of each of the $g_{i}{ }^{\prime}$ s.

Proof. If $u$ is equivalent to $v$, there is a finite sequence of elementary transformations which takes $u$ to $v$. If $u$ and $v$ are identical, the result holds trivially. If there is one elementary transformation taking $u$ to $v$, there is an identity $w\left(x_{1}, \ldots, x_{m}\right)=z\left(x_{1}, \ldots, x_{m}\right)$ among the defining identities for $V$ and a subword $w\left(a_{1}, \ldots, a_{m}\right)$ of $u$, where $a_{1}, \ldots, a_{m}$ are words in the $g_{i}$ 's, such that $v$ is obtained from $u$ by replacing one occurrence of the subword $w\left(a_{1}, \ldots, a_{m}\right)$ in $u$ by the word $z\left(a_{1}, \ldots, a_{m}\right)$. Since $w(\mathbf{x})=z(\mathbf{x})$ is a balanced identity, the words $w(\mathrm{a})$ and $z(\mathrm{a})$ have the same length and number of occurrences of each of the $g_{i}$ 's. Since $u$ and $v$ differ only by these subwords, $|u|_{g}=|v|_{g}$ and each $g_{i}$ occurs the same number of times in $u$ as it occurs in $v$.

The result follows by induction on the number of elementary transformations used in taking $u$ to $v$.

An algebra is said to be bopfian if it is not isomorphic to a proper homomorphic image. We show that the finitely generated free algebras in a balanced variety of finite similarity type are hopfian. An algebra $A$ is residually finite if for any two elements $x$ and $y$ in $A$, there is a homomorphism $\alpha$ of $A$ onto a finite algebra such that $x a \neq y a$. T. Evans has proved that a finitely generated residually finite algebra is hopfian [4]. In [6, p. 19], Evans proves that free semigroups are residually finite. We generalize his argument to prove the following lemma.

Lemma 5. Let $V$ be a balanced variety of finite similarity type and $F$ be the free algebra in $V$ on $g_{1}, g_{2}, \ldots$ Then $F$ is residually finite.

Proof. $F$ consists of equivalence classes of words in the $g_{i}$ 's. We let $[u]_{F}$ denote the class containing the word $u$. Let $u$ and $v$ be words such that $[u]_{F} \neq[v]_{F}$, and let $m$ be the maximum of $|v|_{g}$ and $|u|_{g}$. By Lemma 4, all the words in an equivalence class have the same length and involve the same generators. Define the relation $\theta$ on $F$ as follows: $\left([w]_{F},[z]_{F}\right)$ belongs to $\theta$ if and 
only if $[w]_{F}=[z]_{F}$ or each element of $[w]_{F}$ and $[z]_{F}$ has length greater than $m$ or involves some generator which occurs neither in $u$ nor in $v$. Then $\theta$ is a congruence relation on $F$.

Since the number of operations on $F$ is finite and so is the number of generators that occur in $u$ or $v$, there are only a finite number of words which are of length less than or equal to $m$ and involve only generators which occur in $u$ or $v$. Then $F$ has only a finite number of elements containing words of this form. Since all other elements of $F$ are congruent modulo $\theta, F / \theta$ is a finite homomorphic image of $F$. Clearly, $[u]_{F}$ is not congruent to $[v]_{F}$ modulo $\theta$.

Now applying the Evans result [4] cited above, we have

Lemma 6. Let $V$ be a balanced variety of finite similarity type and $F$ be an algebra which is $V$-free on a finite number of generators. Then $F$ is bopfian.

We now apply the preceding lemmas to prove a theorem like the Whitehead theorem for any balanced variety of finite similarity type.

Theorem 8. Let $V$ be a balanced variety of finite similarity type. Let $A$ be an algebra in $V$ presented by $\left\langle g_{1}, \ldots, g_{n}: r\left(g_{1}, \ldots, g_{n}\right)=s\left(g_{1}, \ldots, g_{n}\right)\right\rangle$, where $r(\mathbf{g})=s(\mathbf{g})$ is a nontrivial relation. Then, $A$ is free if and only if the relation is of the form $g_{i}=w\left(g_{1}, \cdots, g_{i-1}, g_{i+1}, \cdots, g_{n}\right)$, for some word $w$.

Proof. By Theorem 1, we only have to show that, if $A$ is free, the relation is of the right form. In this case, let $F$ be the free algebra in $V$ on $g_{1}, \ldots, g_{n}$. We consider $F$ and $A$ to consist of equivalence classes of words in the $g_{i}$ 's. We use $F$ and $A$ as subscripts to distinguish between elements of and equivalence in $F$ and $A$.

Let $\phi$ be the congruence on $F$ generated by $(r, s)$. Then, $A \cong F / \phi$. Since $r=s$ is a nontrivial relation, $F / \phi$ is a proper homomorphic image of $F$. Suppose that $F / \phi$ is free on $n$ or more generators. Then, a homomorphic image of $F / \phi$ is free on $n$ generators, and $F$ is isomorphic to a proper homomorphic image. This contradicts $F$ being hopfian. Therefore, $A$ is free on $k$ generators, $k \leq n-1$.

We now show that, if neither $r$ nor $s$ is identically $g_{j}$, then $g_{j}$ is the only word in $\left[g_{j}\right]_{A}$. If $u \sim_{A} g_{j}$, there are words $u_{0}, \cdots, u_{m}$ such that $g_{j}=u_{0}, u=u_{m}$, and, for each $i=0,1, \ldots, m, u_{i+1}$ is obtained from $u_{i}$ by applying some elementary transformation in $A$. If some transformation $u_{i} \rightarrow u_{i+1}$ is a use of the relation $r=s$, let $u_{q} \rightarrow u_{q+1}$ be the first such. Then, taking $g_{j}=u_{0}$ to $u_{q}$ requires only the use of elementary transformations involving identities, and $g_{j} \sim_{F} u_{q}$. By Lemma $4, u_{q}$ is identically $g_{j}$. Hence, $u_{q}$ cannot have either $r$ or $s$ as a subword. Thus, $u=u_{m}$ is obtained from $g_{j}$ without using the relation $r=s$ and is identically $g_{j}$. 
Let $w_{1}, \ldots, w_{k}$ be words in $g_{1}, \ldots, g_{n}$ such that $\left[w_{1}\right]_{A}, \ldots,\left[w_{k}\right]_{A}$ freely generate $A$. Now suppose that neither $r$ nor $s$ is one of the $g_{j}$ 's. For each $j=1, \ldots, n,\left[g_{j}\right]_{A}=z_{j}\left(\left[w_{1}\right]_{A}, \ldots,\left[w_{k}\right]_{A}\right)=\left[z_{j}\left(w_{1}, \ldots, w_{k}\right)\right]_{A}$ for some word $z_{j}\left(x_{1}, \ldots, x_{k}\right)$. Since the word $z_{j}\left(w_{1}, \ldots, w_{k}\right)$ is in $\left[g_{j}\right]_{A}$, we must have that $z_{j}\left(x_{1}, \ldots, x_{k}\right)=x_{q}$ for some $q$ and $w_{q}=g_{j}$. This contradicts the fact that there are no more than $n-1$ different $w_{j}$ 's. Thus, one of $r$ or $s$ is some $g_{i}$ and the relation $r=s$ is of the form $g_{i}=w\left(g_{1}, \ldots, g_{n}\right)$.

Suppose $g_{i}$ occurs in $w\left(g_{1}, \cdots, g_{n}\right)$. Then, for each $j \neq i, g_{j}$ is the only word in $\left[g_{j}\right]_{A}$. Following the argument above, each $\left[g_{j}\right]_{A}, j \neq i$, must be among $\left[w_{1}\right]_{A}, \ldots,\left[w_{k}\right]_{A}$. Then, $\left\{\left[g_{j}\right]_{A}: j \neq i\right\}=\left\{\left[w_{j}\right]_{A}: j=1, \ldots, k\right\}$ and $k=n-1$. If $u \sim_{A} g_{i}, u$ is obtained from $g_{i}$ using identities or the relation. Since each side of the relation has an occurrence of $g_{i}, g_{i}$ has an occurrence of $g_{i}$, and the identities are balanced, $u$ must have an occurrence of $g_{i}$. Then, $\left[g_{i}\right]_{A}$ is not generated by $\left\{\left[g_{j}\right]_{A}: j \neq i\right\}$. Since this is a contradiction, the relation is of the form $g_{i}=w\left(g_{1}, \ldots, g_{i-1}, g_{i+1}, \cdots, g_{n}\right)$.

This result is very must like the Whitehead theorem. Using ideas from the proof of Theorem 8 , there is only one free generating set for a free algebra in a balanced variety. Hence, the only primitive elements in the free algebra on $g_{1}, \cdots, g_{n}$ are the elements $g_{1}, \cdots, g_{n}$.

In the introduction we raised a very broad question which is yet to be answered. The results here mainly deal with particular varieties. Numerous interesting problems should arise from considering what theorem can be proved for other particular varieties. Notable among familiar varieties not considered here are the varieties of lattices, associative rings, and nonassociative rings.

\section{REFERENCES}

1. V. D. Belousov, Balanced identities in quasigroups, Mat. Sb. 70 (112) (1966), 55-97. (Russian) MR 34 \#2757.

2. P. M. Cohn, Universal algebra, Harper and Row, New York, 1965. MR 31 \#224.

3. T. Evans, The word problem for abstract algebras, J. London Math. Soc. 26 (1951), 64-71. MR 12, 475.

4. - Finitely presented loops, lattices, etc. are hopfian, J. London Math. Soc. 44(1969), 551-552. MR 38 \#5975.

5. - Schreier varieties of semigroups, Math. Z. 112 (1969), 296-299. MR 40 \#2776.

6. The lattice of semigroup varieties, Semigroup Forum 2 (1971), no. 1, 1-43. MR 44 \# 1753.

7. B. Jonsson and A. Tarski, On two properties of free algebras, Math. Scand. 9 (1961), 95-101. MR 23 \#A3695.

8. G. P. Kukin, Primitive elements of free Lie algebras, Algebra i Logika 9 (1970), 458-472. MR $44 \# 1708$. 
9. A. G. Kuroš, Nonassociative free algebras and free products of algebras, Mat. Sb. 20 (62) (1947), 239-262. (Russian) MR 9, 5.

10. J. Lewin, On Schreier varieties of linear algebras, Trans. Amer. Math. Soc. 132 (1968), 553-562. MR $37 \# 262$.

11. W. Magnus, A. Karrass and D. Solitar, Combinatorial group theory: Presentations of groups in terms of generators and relations, Pure and Appl. Math., vol. 13, Interscience, New York, 1966. MR 34 \#7617.

12. A. I. Širšov, On subalgebras of free Lie algebras, Mat. Sb. 33 (75) (1953), 441452. (Russian) MR 15, 596.

13. - On subalgebras of free commutative and free anticommutative algebras, Mat. Sb. 34 (76) (1954), 81-88. (Russian) MR 15, 929.

14. J. H. C. Whitehead, On equivalent sets of elements in a free group, Ann. of Math. 37 (1936), 782-800.

15. E. Witt, Die Unterringe der freien Lieschen Ringe, Math. Z. 64 (1956), 195-216. MR 17, 1050.

DEP ARTMENT OF MATHEMATICS, GEORGIA INSTITUTE OF TECHNOLOGY, ATL ANTA, GEORGIA 30332 (Current address)

DEPARTMENT OF MATHEMATICS, EMORY UNIVERSITY, ATLANTA, GEORGIA 30332 